\title{
Safety and Efficiency of Biomimetic Nanohydroxyapatite/Polyamide 66 Composite in Rabbits and Primary Use in Anterior Cervical Discectomy and Fusion
}

\author{
Hui Xu, ${ }^{1,2}$ Yan Wang, ${ }^{2}$ Xiaojing Su, ${ }^{2}$ Xuelian Zhang, ${ }^{3}$ and Xuesong Zhang ${ }^{2}$ \\ ${ }^{1}$ Department of Orthopedics, Liaocheng People’s Hospital, 67 Dongchang West Road, Liaocheng, Shandong 252004, China \\ ${ }^{2}$ Department of Orthopedics, Chinese People's Liberation Army General Hospital, Beijing 100853, China \\ ${ }^{3}$ Department of Endocrinology, China-Japan Friendship Hospital, Beijing 100029, China \\ Correspondence should be addressed to Xuesong Zhang; zhangxuesong301@sina.com
}

Received 6 March 2014; Accepted 4 May 2014; Published 4 June 2014

Academic Editor: Xiaoming Li

Copyright (C) 2014 Hui Xu et al. This is an open access article distributed under the Creative Commons Attribution License, which permits unrestricted use, distribution, and reproduction in any medium, provided the original work is properly cited.

\begin{abstract}
This study was conducted to validate the safety and efficiency of biomimetic nanohydroxyapatite/polyamide 66 (n-HA/PA66) composite in animal model (rabbit) and report its application in anterior cervical discectomy and fusion (ACDF) for 4, 12, and 24 weeks. N-HA/PA66 composite was implanted into one-side hind femur defects and the control defects were kept empty as blank controls. A combination of macroscopic and histomorphometric studies was performed up to 24 weeks postoperatively and compared with normal healing. 60 cervical spondylosis myelopathy and radiculopathy patients who were subjected to ACDF using n-HA/PA66 and PEEK cage were involved in this study with six-month minimum follow-up. Their radiographic (cage subsidence, fusion status, and segmental sagittal alignment (SSA)) and clinical (VAS and JOA scales) data before surgery and at each follow-up were recorded and compared. Nanohydroxyapatite/polyamide 66 composite is safe and effective in animal experiment and ACDF.
\end{abstract}

\section{Introduction}

Generally, ideal bone graft substitute materials should have good osteogenesis, biocompatibility, and bioactivity. Furthermore, they can provide enough mechanical strength to meet the fundamental support requirements during bone healing period. Based on that concept, more attention is focused on the development of bioactive composite composed of bioactive inorganic ingredient (osteogenesis) and flexible polymer in recent years. Hydroxyapatite (HA) shows a similar composition and structure to natural bone mineral and therefore has been considered to be an ideal material to build bone repair materials due to its osteoconductivity. But its brittleness extremely limits its use in load-bearing bone repair [1-7]. Polyamide (PA) has good biocompatibility probably because of its similarity to collagen protein in chemical structure and active groups $[8,9]$. PA also has excellent mechanical properties resulting from the strong hydrogen bonds between the amide groups in PA macromolecules [10, 11].
To combine advancements of the two materials, nanohydroxyapatite (n-HA)/polyamide 66 biocomposite was developed by Sichuan University [12]. This composite showed good mechanical strength similar to natural bone and good compatibility. Furthermore, cage and vertebral plate developed by this composite have been used for spine repair successfully [13-18]. The porous n-HA/PA66 composite had biological safety, good biocompatibility, osteoinduction, and osseointegration $[19,20]$. However, most of the studies were conducted in one institution. The purpose of this study is to validate the safety and efficiency of biomimetic n-HA/PA66 composite in rabbits and report primary use in cervical discectomy and fusion in our department.

\section{Materials and Method}

\subsection{Animal Experiment}

2.1.1. n-HA/PA66 Characterization. The n-HA/PA66 biocomposite was prepared by thermal-press molding. The 


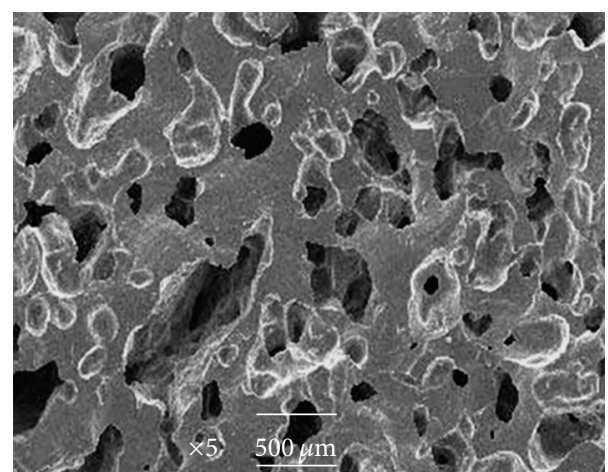

FIgURE 1: SEM micrographs of porous n-HA/PA66.

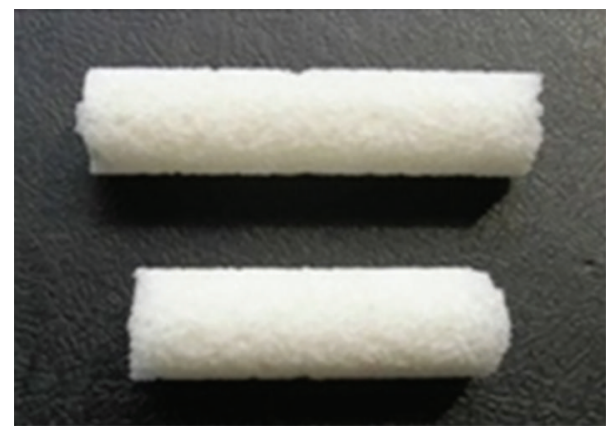

FIGURE 2: Columnar n-HA/PA blocks.

material presented: (1) high compressive strength which reached $13 \sim 46 \mathrm{MPa}$ and (2) excellent porous structure; the average diameter of pores in the matrix was in range of $280 \mu \mathrm{m}$ to $500 \mu \mathrm{m}$ and porosity of $36 \%$ to $57 \%$ (Figure 1) [21]. The columnar n-HA/PA blocks (height $10 \mathrm{~mm}$ and diameter $5 \mathrm{~mm}$ ) were made by Sichuan National Nano Technology Co., Ltd. (Chengdu, China) (Figure 2). The biocomposite was sterilized by gamma radiation prior to implantation.

2.1.2. n-HA/PA66 Implantation. 30 New Zealand white rabbits were involved in this experiment. After anesthesia, about $5 \mathrm{~cm}$ longitudinal incision was made $1 \mathrm{~cm}$ higher to the hind limb knee to expose the femur. Four defects $(5 \mathrm{~mm} * 5 \mathrm{~mm})$ were drilled at low speed in the femur. Then the n-HA/PA 66 blocks were inserted into the defects in two of four bone defects and the other two defects were kept empty. Implant placement was randomized in the four defects and the wound was secured in layers. After surgery the rabbits were randomly divided into 3 groups according to sacrifice time $(4,12$, and 24 weeks).

All animals were injected with 10,000 units of penicillin per day for 3 days to prevent infection. The animals were sacrificed by a lethal dose of barbiturate, respectively, at 4,12 , or 24 weeks after surgery. The femurs were removed, cleaned, and prepared for histologic testing.

2.1.3. Wound Healing and Local Inflammatory Reaction. Swelling, hematoma, infection, wound healing, and associated signs of local inflammatory reactions were observed

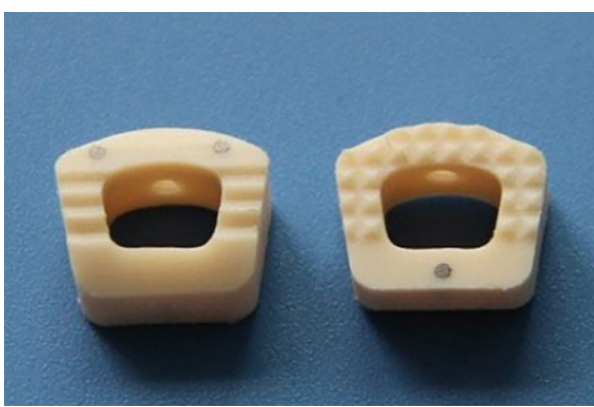

Figure 3: The n-HA/PA66 cage.

every day in 1 week after surgery and once in 1 week up to 24 weeks postoperatively.

2.1.4. Macroscopic Observations and Histological Study. At the time of 4,12 , and 24 weeks after surgery, the rabbits were killed and the femurs were explored. Local bone healing was observed. $5 \mathrm{~cm}$ femur samples including all the defects were removed with a diamond saw. The bone samples were cleaned and were doused in $10 \%$ formalin for 7 days. After initial fixation, the bones were dehydrated in a series of alcohol until the standardization was reached. Then the samples were decalcified and embedded in epoxy resin. $5 \mu \mathrm{m}$ cross-sections were cut by a diamond saw microtome (Leica Spa, Milan, Italy), and Masson's trichrome staining was conducted. The stained sections of each sample were observed with a light microscope (NICON E 600).

\subsection{Primary Clinical Use}

2.2.1. Patients and Methods. This was a prospective nonrandom compared study of patients with cervical spondylosis myelopathy or radiculopathy who underwent anterior cervical discectomy and fusion (ACDF) with cage and anterior plate. The study was approved by the Institutional Review Board and was performed at our department from January 2013 to June 2013.

During this period, 60 patients were eligible for inclusion. The inclusion criteria were (1) myelopathy or radiculopathy with failure of conservative treatment (of at least three months), (2) cervical degenerative diseases with compression of the cervical spinal cord or nerve roots, or best clinical symptoms and signs which can explain well documented radiographs, and (3) through ACDF surgery, the spinal cord or nerve roots could be adequately decompressed. The exclusion criteria were (1) cervical deformities or neoplasia, (2) severe osteoporosis, and (3) patients with psychiatric disorders. The clinical presentation and radiological findings determined the level of discectomy. The anterior fusion was performed using n-HA/PA66 cage (Sichuan National Nano Technology Co., Ltd., Chengdu, china) (see Figure 3) in 30 patients and PEEK cage (Medtronic Sofamor Danek Inc., Memphis, TN) in the other patients. 
All the operations were performed by one of two senior spine surgeons. The patients were placed in the supine position with neck extension. Cervical discectomy and decompression were performed in the standard right-side anterior cervical approach. Then local autologous bone filling PEEK or n-HA/PA66 cage was implanted into the disc space. The Atlantis anterior cervical plate system (Medtronic Sofamor Danek Inc., Memphis, TN) was used in each patient for further stabilization. After surgery, all patients were requested to wear a soft cervical collar for approximately four weeks.

2.2.2. Outcomes Assessment. At three and six months after surgery, the patients had radiological follow-up. The cervical plain radiographs were taken at each follow-up examination. The following parameters were evaluated on lateral plain radiographs: preoperative and postoperative segmental sagittal alignment (SSA), cage subsidence, and fusion status. The height of the fusion segment was measured as the distance between the midpoint of the superior endplate of the upper vertebra and the midpoint of the inferior endplate of the lower vertebra. Subsidence was defined as the difference of height of the fusion segment between immediately after surgery and the later follow-up. It would be considered as severe subsidence once cage subsidence exceeded three millimeters. We defined the Cobb angle between the superior endplate of the C4 vertebra and the inferior endplate of the C7 vertebra as the SSA. The lordosis angle and the kyphosis angle were defined as positive angle and negative angle, respectively. The changes in sagittal alignment before surgery and at postoperative follow-ups were determined. When a trabecular bone across the interfaces appeared, fusion was considered complete. If the lucency between the implants and vertebral endplates surfaced, nonunion would be noted. All these radiographic parameters were measured by five independent senior spine surgeons who were not involved in the primary surgery and were unaware of the clinical results of the patients. Their average value was adopted for final analysis.

In addition, the clinical outcome of each patient was assessed by comparing the scores on a 10-point visual analogue scale (VAS) and the Japanese Orthopedic Association (JOA) scale before surgery and at six-month follow-up. Excessive operation time, blood loss, hospital stay, and surgical complications were recorded. The recovery rate (JOA) was measured by the method of Hirabayashi et al. [22]. Recovery rate $(\%)=$ post-op score - pre-op score/17 (full) - pre-op score $\times 100$.

2.3. Statistical Analysis. In this study, SPSS 17.0 statistic software (SPSS, Chicago, IL, USA) was used for all statistical analyses. Quantitative data are presented as the mean \pm standard deviation. With one-way ANOVA, the mean comparison at different time points was conducted, and with the LSD method the pairwise comparison was performed. By the Mann-Whitney test, mean comparison in two groups was analyzed. All $P$ values were two-sided, and levels of significance reaching $>95 \%$ were accepted.

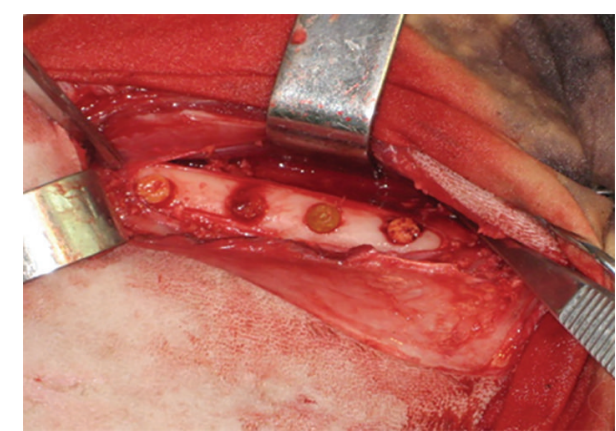

Figure 4: 4 weeks after implanting n-HA/PA66 blocks.

\section{Result}

\subsection{Animal Experiments}

3.1.1. Wound Healing and Local Inflammatory Reactions. All the rabbits survived until they were killed. Wounds healed well in all cases and sutures were removed on the tenth postoperative day. There was no swelling, fluid, or local heat during the postoperative period.

3.1.2. Macroscopic Observations. At the time of 4, 12, and 24 weeks after surgery, the femurs were exposed and were macroscopically observed. There were no obvious signs of local inflammation observed in any animal. At the fourth week, the implants were stable in the defects and the callus formation covered about $70 \%$ of the surface of implants (Figure 4); in the blank control defects, few calluses were observed. At the twelfth week, the whole surface of the implant was almost covered by callus. But in the blank control, the calluses were fewer. At twentieth week, the defects in the n-HA/PA 66 group were covered with the natural bone. In the control group, the callus was thin and the defects were still visible.

3.1.3. Histological Analyses. The defects with n-HA/PA implantation in the 4-week group were filled with newly formed bone and connective tissue. The interface between the implant and bone was filled with direct bone bonding. New osteoid tissue was observed in the pore structure, and osteoblast infiltration was seen on the surface of the implant. In the control group, loose fibrocollagen tissue filled the defects and no bone formation was observed. In the 12-week group, large amounts of new bone tissue are invading the porosity of the n-HA/PA 66 implant. Osteoid tissue was also detected in the central area of the implant. The lamellar bone transition was seen in formed bone area. In the control group, although new bone tissue was observed, fibrous tissue was more frequently observed. New bony tissue was only detected in marginal areas. 24 weeks postoperatively, part of n-HA/PA 66 also remained. But the cortex of the bone showed welldeveloped lamellar appearance. Mature bone trabecular was seen in no implant defects, but it was only good in marginal areas of the defects. 


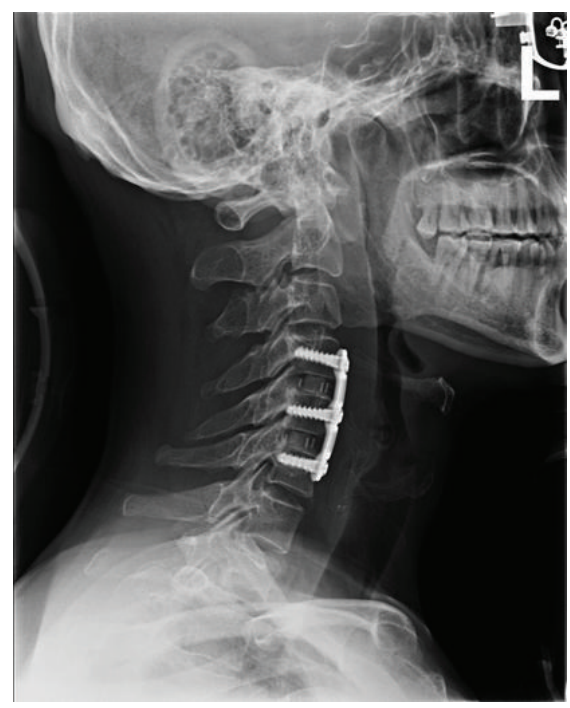

FIGURE 5: PEEK cages in a 65-year-old male postoperatively.

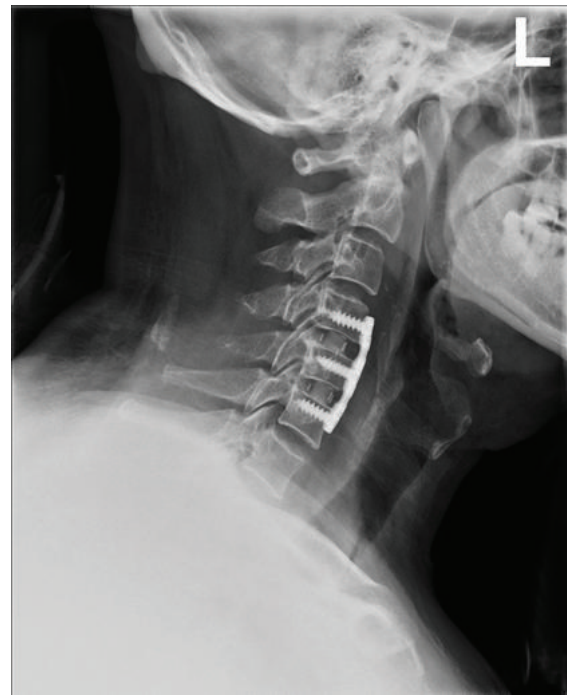

FiguRE 6: n-HA/PA66 cages in a 62-year-old male postoperatively.

3.2. Clinical Results. Of the 60 patients eligible for inclusion in this study, we obtained complete datasets (preoperative, immediately postoperative, 3 months postoperative, and 6month follow-up data) for 57 patients (95\%). It contained 37 men and 20 women with a mean age of 51.3 years (range 35-71 years). 29 patients underwent the ACDF with PEEK cage (PEEK group) (Figure 5) and the other 28 patients with n-HA/PA66 cage (n-HA/PA66 group) (Figure 6). The demographics of both groups of patients are shown in Table 1. There were no statistically significant differences in sex, age, smoking, diagnosis, or pathogenic level between these two groups (all $P>0.05$ ). There were no severe complications in both groups.

3.2.1. Radiographic Analysis. There was no case of cage migration or breakage in either group at the last follow-up.
TABLE 1: Demographic data of cervical spondylitis.

\begin{tabular}{lcc}
\hline & $\begin{array}{c}\text { PEEK group } \\
(n=30)\end{array}$ & $\begin{array}{c}\text { n-HA/PA66 group } \\
(n=30)\end{array}$ \\
\hline M: F & $18: 12$ & $19: 11$ \\
One level & 22 & 24 \\
Two levels & 8 & 6 \\
C4-C5 & 9 & 10 \\
C5-C6 & 18 & 17 \\
C6-C7 & 11 & 9 \\
Radiculopathy & 8 & 9 \\
Myelopathy & 16 & 14 \\
Radiculomyelography & 6 & 7 \\
\hline
\end{tabular}

There are no statistical differences in each item between the groups.

TABLE 2: Clinical outcomes.

\begin{tabular}{lcc}
\hline & $\begin{array}{c}\text { PEEK group } \\
(n=29)\end{array}$ & $\begin{array}{c}\text { n-HA/PA66 group } \\
(n=28)\end{array}$ \\
\hline \multicolumn{3}{c}{ VAS scores of the two groups } \\
Pre-op & $7.1 \pm 1.4$ & $7.3 \pm 1.6$ \\
Six-month follow-up & $2.1 \pm 1.3$ & $2.3 \pm 1.2$ \\
\hline \multicolumn{3}{c}{ JOA scores of the two groups } \\
Pre-op & $8.4 \pm 2.4$ & $8.2 \pm 1.6$ \\
Six-month follow-up & $15.1 \pm 1.6$ & $15.6 \pm 1.2$ \\
\hline
\end{tabular}

There are no statistical differences in each item between the groups.

At three months after surgery, 24 patients' cages were fused with a fusion rate of $83 \%$ in the PEEK group that was markedly lower than the fusion rate of $93 \%$ (26 of 28 ) in the n-HA/PA66 group. At the six-month follow-up, the fusion rate was $100 \%$ in the two groups. There was no severe cage subsidence in the two groups. In the PEEK group, the mean SSA before surgery was $13.8^{\circ}$, and it significantly improved to $19.6^{\circ}$ immediately after surgery $(P<0.05)$. While in the $\mathrm{n}$ HA/PA66 group, the mean preoperative SSA was $14.1^{\circ}$, and it significantly improved to $19.8^{\circ}$ immediately after surgery $(P<0.05)$. The change of SSA before and after surgery was similar in the PEEK group and n-HA/PA66 group. In later follow-ups, the mean SSA of the PEEK group and n-HA/PA66 group had no significant change.

Clinical Outcome. The six-month follow-up VAS and JOA scores of the two groups were significantly improved compared to preoperative scores. However, there is no statistical difference between the two groups over the follow-up period (Table 2).

\section{Discussion}

Bone grafts are widely used in orthopedics surgery and should provide support, bone conduction, and induction. Autogenous bone grafts are considered the gold standard in bone transplantation, but donor sites complications concern many surgeons. Thus biological substitutes were developed by many researchers. Developments of bone substitutes are 
based on a better understanding of the biological process in the bone healing. The biological approach provides the key that plays an important role in the repair of bone defects $[23,24]$. Artificial bone substitutes are clearly inferior to autogenous bone grafts in osteoinduction, but they enhance osteoconduction, provide support, and can be easily made in the required shapes. Another important point which should be considered is histocompatibility.

The n-HA/PA 66 used in this study was prepared by thermal-press molding. Compared to injection molding $\mathrm{HA} / \mathrm{PA}$ [18], the pores in the materials prepared by the hot-press molding are irregular tubular, the connectivity of which is good. The shape and connectivity of the pores in the biomaterials have important effects on their biological activities in vitro and in vivo [21]. The irregular tubular biomaterial provide good interface between n-HA/PA 66 and host bone; new bone tissue can survive in the pores. The compressive strength of n-HA/PA 66 used in this study was 13 46 Mpa. N-HA/PA 66 can provide enough support during the bone healing period.

In this study, n-HA/PA 66 was well accepted and tolerated by all the rabbits, no infection, wound complications, serious inflammation, and rejection response were found in any case. The results of macroscopic and histologic analysis proved better bone reparation in the n-HA/PA 66 group than the blank controls in all groups. New bone tissue was detected around the implant and inside the pore structure 4 weeks postoperatively, which strongly demonstrated the good osteoconductivity of the bone substitute.

In our clinical study, the cage was made of n-HA/PA 66 , the same material used in the animal study. Compared to the traditional PEEK cage used in ACDF, n-HA/PA 66 cages had the higher fusion rate at 3-month follow-up, but at 6-month follow-up, there was no difference. The operation time, blood loss, complications, and clinical outcome scores were similar in the two groups. The results were similar to other reports $[25,26]$. However, the follow-up period was short and the probability of similar case was little. Further study is necessary.

In conclusion, the n-HA/PA 66 composite was safe and effective in repairing bone defects of rabbits. The excellent osteogenesis of the porous n-HA/PA 66 composite in this study was rationalized by not only the biomimetic composition but also the interconnecting porosity. Porous n-HA/PA 66 cage was successfully used in ACDF and had a good outcome that it can be a substitution to traditional cages.

\section{Conflict of Interests}

The authors have no conflict of interests.

\section{References}

[1] G. Wei and P. X. Ma, "Structure and properties of nanohydroxyapatite/polymer composite scaffolds for bone tissue engineering," Biomaterials, vol. 25, no. 19, pp. 4749-4757, 2004.

[2] X. Li, Q. Feng, X. Liu, W. Dong, and F. Cui, “Collagen-based implants reinforced by chitin fibres in a goat shank bone defect model," Biomaterials, vol. 27, no. 9, pp. 1917-1923, 2006.
[3] X. Li, C. A. van Blitterswijk, Q. Feng, F. Cui, and F. Watari, “The effect of calcium phosphate microstructure on bone-related cells in vitro," Biomaterials, vol. 29, no. 23, pp. 3306-3316, 2008.

[4] P. X. Ma, R. Zhang, G. Xiao, and R. Franceschi, "Engineering new bone tissue in vitro on highly porous poly (alpha-hydroxyl acids)/hydroxyapatite composite scaffolds," Journal of Biomedical Materials Research, vol. 54, pp. 284-293, 2001.

[5] J. Wei, Y. Li, and K.-T. Lau, "Preparation and characterization of a nano apatite/polyamide6 bioactive composite," Composites B: Engineering, vol. 38, no. 3, pp. 301-305, 2007.

[6] R. Detsch, H. Mayr, and G. Ziegler, "Formation of osteoclastlike cells on HA and TCP ceramics," Acta Biomaterialia, vol. 4, no. 1, pp. 139-148, 2008.

[7] M. Motskin, D. M. Wright, K. Muller et al., "Hydroxyapatite nano and microparticles: correlation of particle properties with cytotoxicity and biostability," Biomaterials, vol. 30, no. 19, pp. 3307-3317, 2009.

[8] Y.-W. Wang, Q. Wu, J. Chen, and G.-Q. Chen, "Evaluation of three-dimensional scaffolds made of blends of hydroxyapatite and poly(3-hydroxybutyrate-co-3-hydroxyhexanoate) for bone reconstruction," Biomaterials, vol. 26, no. 8, pp. 899-904, 2005.

[9] H. H. K. Xu and C. G. Simon Jr., "Fast setting calcium phosphate-chitosan scaffold: mechanical properties and biocompatibility," Biomaterials, vol. 26, no. 12, pp. 1337-1348, 2005.

[10] S.-S. Kim, M. Sun Park, O. Jeon, C. Yong Choi, and B.-S. Kim, "Poly(lactide-co-glycolide)/hydroxyapatite composite scaffolds for bone tissue engineering," Biomaterials, vol. 27, no. 8, pp. 1399-1409, 2006.

[11] S. I. Lee and B. C. Chun, "Mechanical properties and fracture morphologies of poly(phenylene sulfide)/nylon66 blendseffect of nylon66 content and testing temperature," Journal of Materials Science, vol. 35, no. 5, pp. 1187-1193, 2000.

[12] X. Wang, Y. Li, J. Wei, and K. De Groot, "Development of biomimetic nano-hydroxyapatite/poly(hexamethylene adipamide) composites," Biomaterials, vol. 23, no. 24, pp. 47874791, 2002.

[13] X. Yang, Y. Song, L. Liu, H. Liu, J. Zeng, and F. Pei, "Anterior reconstruction with nano-hydroxyapatite/polyamide-66 cage after thoracic and lumbar corpectomy," Orthopedics, vol. 35, no. 1, pp. e66-e73, 2012.

[14] Z. Zhao, D. Jiang, Y. Ou, K. Tang, X. Luo, and Z. Quan, "A hollow cylindrical nano-hydroxyapatite/polyamide composite strut for cervical reconstruction after cervical corpectomy," Journal of Clinical Neuroscience, vol. 19, no. 4, pp. 536-540, 2012.

[15] M. Huang, J. Feng, J. Wang, X. Zhang, Y. Li, and Y. Yan, "Synthesis and characterization of nano-HA/PA66 composites," Journal of Materials Science: Materials in Medicine, vol. 14, no. 7, pp. 655-660, 2003.

[16] Q. Xu, H. Lu, J. Zhang, G. Lu, Z. Deng, and A. Mo, “Tissue engineering scaffold material of porous nanohydroxyapatite/polyamide 66," International Journal of Nanomedicine, vol. 5, no. 1, pp. 331-335, 2010.

[17] H. Wang, Y. Li, Y. Zuo, J. Li, S. Ma, and L. Cheng, "Biocompatibility and osteogenesis of biomimetic nano-hydroxyapatite/ polyamide composite scaffolds for bone tissue engineering," Biomaterials, vol. 28, no. 22, pp. 3338-3348, 2007.

[18] J. C. Zhang, H. Y. Lu, G. Y. Lv, A. C. Mo, Y. G. Yan, and C. Huang, "The repair of critical-size defects with porous hydroxyapatite/polyamide nanocomposite: an experimental study in rabbit mandibles," International Journal of Oral and Maxillofacial Surgery, vol. 39, no. 5, pp. 469-477, 2010. 
[19] X. Luo, L. Zhang, Y. Morsi et al., "Hydroxyapatite/polyamide66 porous scaffold with an ethylene vinyl acetate surface layer used for simultaneous substitute and repair of articular cartilage and underlying bone," Applied Surface Science, vol. 257, no. 23, pp. 9888-9894, 2011.

[20] Y. Xiong, C. Ren, B. Zhang et al., "Analyzing the behavior of a porous nano-hydroxyapatite/polyamide 66 (n-HA/PA66) composite for healing of bone defects," International Journal of Nanomedicine, vol. 9, pp. 485-494, 2014.

[21] H. Li, Y. Li, Y. Yan, J. Li, A. Yang, and H. Xiang, "Fabrication of porous n-HA/PA66 composite for bone repair," Key Engineering Materials, vol. 330-332, pp. 321-324, 2007.

[22] K. Hirabayashi, J. Miyakawa, K. Satomi, T. Maruyama, and K. Wakano, "Operative results and postoperative progression of ossification among patients with ossification of cervical posterior longitudinal ligament," Spine, vol. 6, no. 4, pp. 354364, 1981.

[23] X. Li, L. Wang, Y. Fan, Q. Feng, F.-Z. Cui, and F. Watari, "Nanostructured scaffolds for bone tissue engineering," Journal of Biomedical Materials Research A, vol. 101, no. 8, pp. 24242435, 2013.

[24] X. Li, H. Liu, X. Niu et al., "The use of carbon nanotubes to induce osteogenic differentiation of human adipose-derived MSCs in vitro and ectopic bone formation in vivo," Biomaterials, vol. 33, no. 19, pp. 4818-4827, 2012.

[25] F. Suetsuna, T. Yokoyama, E. Kenuka, and S. Harata, "Anterior cervical fusion using porous hydroxyapatite ceramics for cervical disc herniation: a two-year follow-up," The Spine Journal, vol. 1, no. 5, pp. 348-357, 2001.

[26] X. Yang, Q. Chen, L. Liu et al., "Comparison of anterior cervical fusion by titanium mesh cage versus nano-hydroxyapatite/ polyamide cage following single-level corpectomy," International Orthopaedics, vol. 37, no. 12, pp. 2421-2427, 2013. 

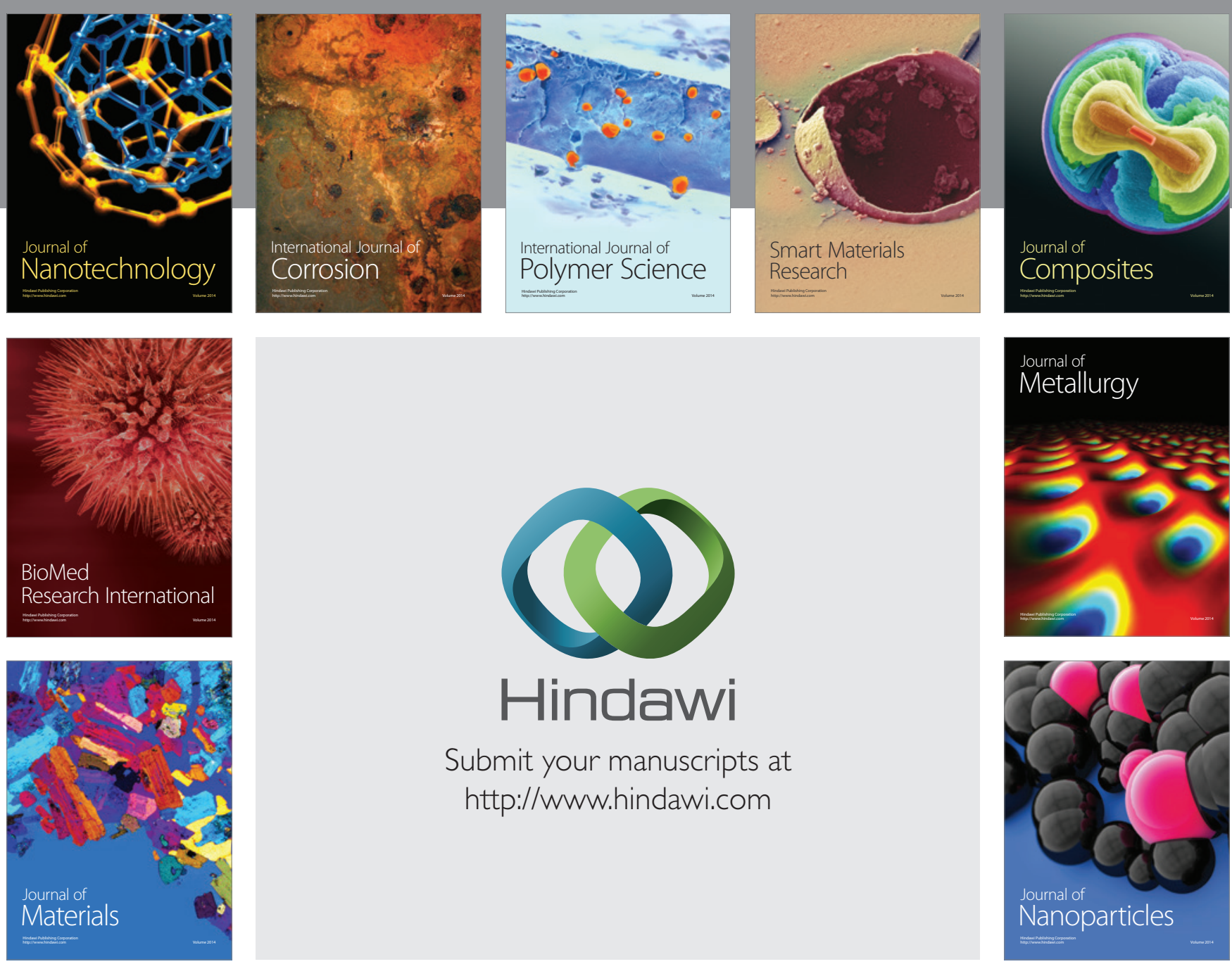

Submit your manuscripts at http://www.hindawi.com
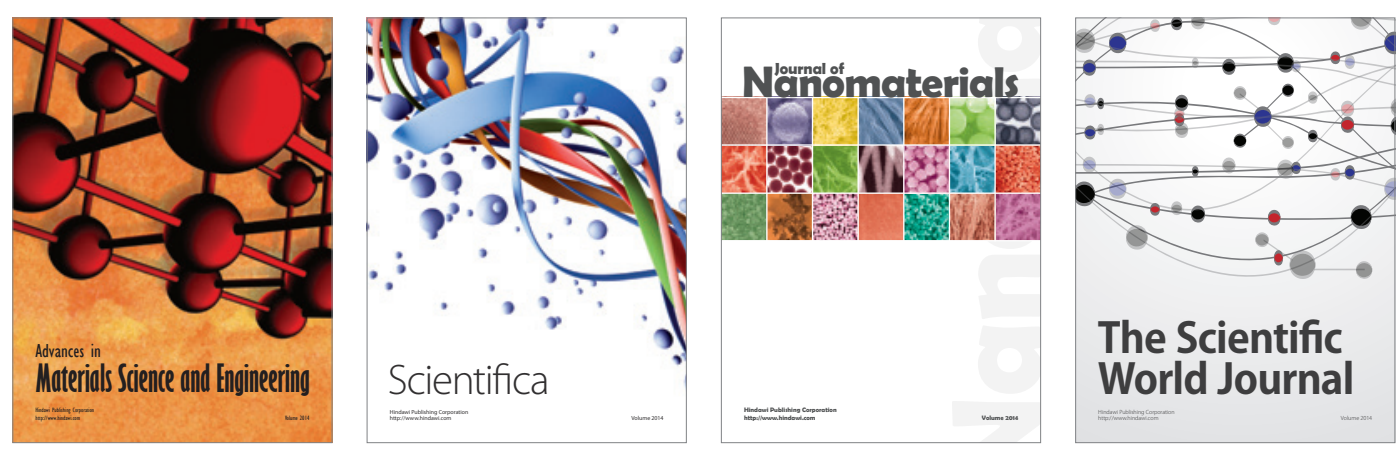

\section{The Scientific World Journal}
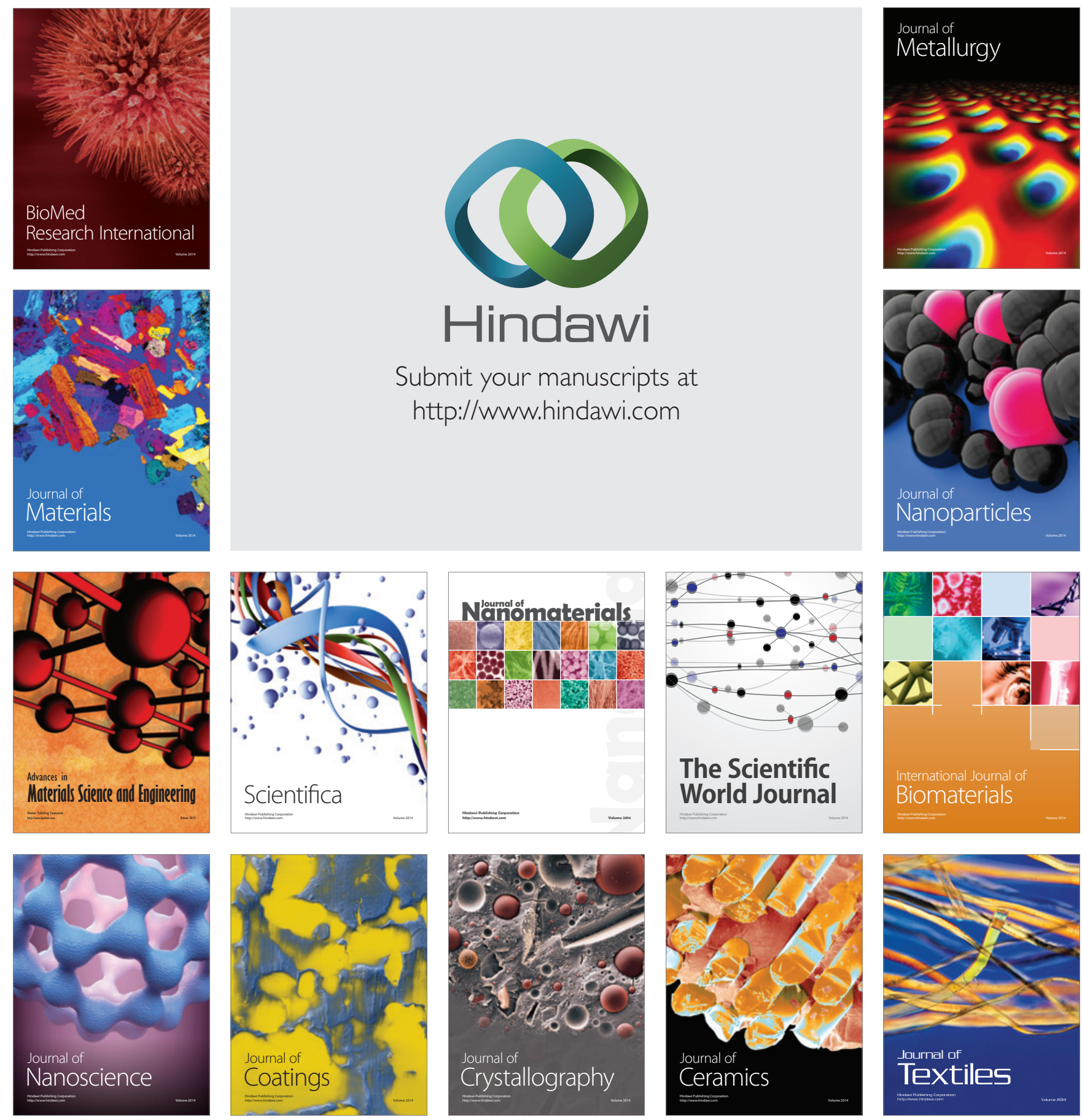